\title{
Ising antiferromagnet with ultracold bosonic mixtures confined in a harmonic trap
}

\author{
M. Guglielmino, ${ }^{1,2}$ V. Penna, ${ }^{3}$ and B. Capogrosso-Sansone ${ }^{2}$ \\ ${ }^{1}$ Dipartimento di Fisica, Politecnico di Torino, Corso Duca degli Abruzzi 24, I-10129 Torino, Italy \\ ${ }^{2}$ Institute for Theoretical Atomic, Molecular and Optical Physics, \\ Harvard-Smithsonian Center of Astrophysics, Cambridge, MA, 02138 \\ ${ }^{3}$ Dipartimento di Fisica and CNISM Unità di Ricerca, \\ Politecnico di Torino, Corso Duca degli Abruzzi 24, I-10129 Torino, Italy
}

\begin{abstract}
We present accurate results based on Quantum Monte Carlo simulations of two-component bosonic systems on a square lattice and in the presence of an external harmonic confinement. Starting from hopping parameters and interaction strengths which stabilize the Ising antiferromagnetic phase in the homogeneous case and at half integer filling factor, we study how the presence of the harmonic confinement challenge the realization of such phase. We consider realistic trapping frequencies and number of particles, and establish under which conditions, i.e. total number of particles and population imbalance, the antiferromagnetic phase can be observed in the trap.

PACS numbers: $67.60 . \mathrm{Bc}, 67.85 . \mathrm{Hj}, 67.85 . \mathrm{Fg}$
\end{abstract}

In recent years great attention has been devoted to mixtures of ultracold atomic gases in optical lattices [18. One of the most remarkable features of such systems is the possibility of stabilizing quantum magnetic phases 9 , 10. Though interesting and fundamental on their own, better understanding of magnetic phases is further motivated by applications to quantum-information processing and their relevance to unconventional superconductivity such as high-temperature and heavy-fermion superconductivity. It has been proposed [11] (and recently experimentally investigated [12]) that antiferromagnetic excitations may be responsible for the pairing mechanism in unconventional superconductors. The experimental realization of magnetic Hamiltonians with ultracold atoms would open up the way for direct control over interactions, geometry, and frustration. Recently an Ising antiferromagnetic phase has been realized with singlecomponent ultracold atoms trapped in a one-dimensional optical lattice tilted by a magnetic field 13 (Ising density wave order could also be realized at lower energy scales in specific two-dimensional tilted geometries [14]).

Magnetic phases with mixtures of ultracold atoms can be realized at low temperatures, strong interactions and specific filling factors. Experimentally, though, the presence of the external confinement will challenge their realization. Previous theoretical studies, focused on BoseBose mixtures, reported accurate results for the groundstate phase boundaries of the Ising antiferromagnetic and the $x y$-ferromagnetic phases at half-filling factor [15], and for the critical temperature needed for their realization [16. The temperature scale, determined by spinexchange interactions, results challengingly low $(\sim$ few hundreds pK). Nonetheless, recent experimental efforts have been devoted towards the development of new techniques of refrigeration and thermometry [7, 8].

The next crucial question to theoretically address is studying the effect of an external trapping potential. In the present work we focus on the Ising antiferromagnetic (AF) phase realized by Bose-Bose mixtures in the strongly interacting regime and at half-filling factor, and study under which conditions it survives the presence of a harmonic confinement. In the local-density approximation, the latter provides a scan over chemical potentials of the two components. Therefore, even if Hamiltonian parameters are experimentally tuned to stabilize the Ising $\mathrm{AF}$ phase in the homogeneous system, the presence of an external confinement will challenge its realization. In other words, other phases such as Mott insulator (MI) + superfluid, double superfluid and phase separation will compete, moreover given the pronounced asymmetry in hopping parameters required for stabilization of the $\mathrm{AF}$ phase [9, 10, 15]. Hence, for a given harmonic confinement, the challenge consists in tuning the particle numbers so that there exist an extended region in the trap with half filling for both components.

In the following we present first precise results based on Quantum Monte Carlo simulations of a two-dimensional system with numbers of particles typical of current experiments 17, 18 and trapping frequencies easily realizable with magnetic or optical traps. We show that observation of the AF phase in a trap is non trivial, but indeed possible, provided one properly tunes the total number of particles and the population imbalance. Hence, our results provide an accurate guidance towards the experimental realization of the Ising AF with mixtures.

We consider a system of two-component bosons in a square lattice, with repulsive interspecies interaction and in the presence of an external harmonic confinement. This system can be realized by loading an optical lattice with two different atomic species [5], or the same atomic species in two different internal energy states 6 8. If intraspecies interactions are much larger than any other energy scale (a limit which can be achieved by, e. g., using Feshbach resonance), and the temperature is low enough, the system is accurately described by the two-component hard-core Bose-Hubbard Hamiltonian:

$H=-\sum_{\langle i j\rangle}\left(t_{a} a_{i}^{\dagger} a_{j}+t_{b} b_{i}^{\dagger} b_{j}\right)-\sum_{\alpha, i} \mu_{i}^{\alpha} n_{i}^{\alpha}+U_{a b} \sum_{i} n_{i}^{a} n_{i}^{b}$,

where $\alpha=a, b$ denotes the two species; $\sum_{\langle i j\rangle}$ is intended 

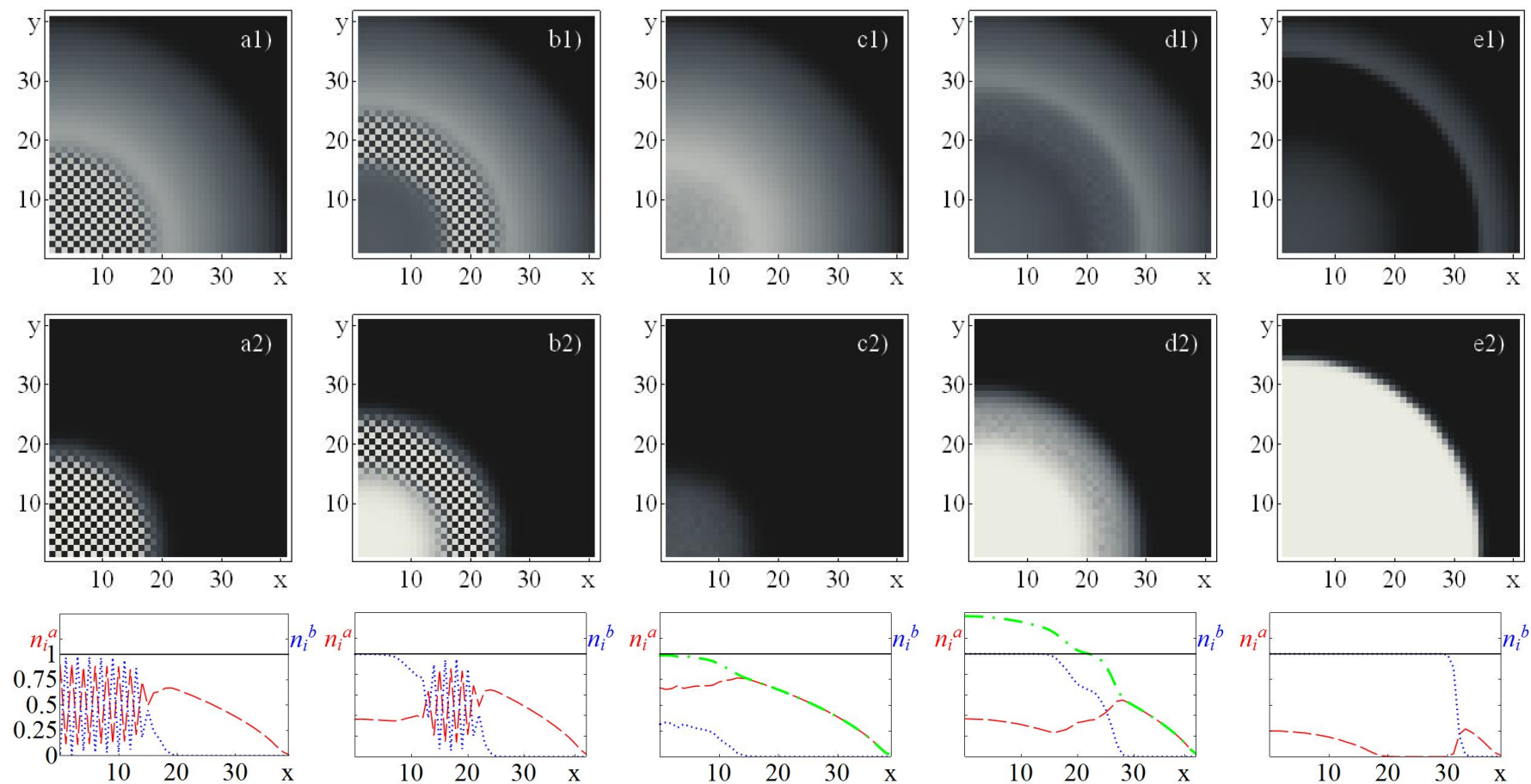

FIG. 1: (Color online) Top and middle rows: representative density maps (light and heavy component respectively) for each type of phase coexistence observed. Color scheme: white (black) corresponds to 1 (0) density. Bottom row: density profiles along a line going through the trap center (dashed red, dotted blue and dot-dashed green lines correspond to light component, heavy component and total density profiles, respectively). Coordinates $x$ and $y$ are given in units of $d$. Hamiltonian parameters: $t_{b}=0.15 t_{a}, U_{a b}=5.7 t_{a}, T=0.05 t_{a}, w=0.005 t_{a}$. First column: $2 \mathrm{CB}$ core, $N_{a}=1940, N_{b}=430$; second column: $2 \mathrm{CB}$ ring, $N_{a}=2155, N_{b}=1030$; third column: global Mott core, $N_{a}=2130, N_{b}=125$; fourth column: global Mott ring, $N_{a}=1750$, $N_{b}=1750$; fifth column: phase separation, $N_{a}=425, N_{b}=3185$.

on nearest-neighboring sites; $a_{i}\left(a_{i}^{\dagger}\right), b_{i}\left(b_{i}^{\dagger}\right)$ are bosonic annihilation (creation) operators at site $i$, satisfying the hard-core constraint; $t_{a}, t_{b}$ are hopping amplitudes; $n_{i}^{a}=a_{i}^{\dagger} a_{i}, n_{i}^{b}=b_{i}^{\dagger} b_{i}$, are number operators; $U_{a b}$ is the interspecies interaction; $\mu_{i}^{\alpha}=\mu_{\alpha}-w\left(\mathbf{r}_{i} / d\right)^{2}$, with $\mu_{\alpha}$ the chemical potential, $\mathbf{r}_{\mathbf{i}}$ the vector position of site $i, d$ the lattice spacing, and $w$ the curvature of the external potential. The usual intraspecies interaction term $H_{\text {int }}=\sum_{\alpha i} U_{\alpha} n_{i}^{\alpha}\left(n_{i}^{\alpha}-1\right) / 2$ has been dropped because the $U_{\alpha} \rightarrow \infty$ limit is considered. In the following we use $t_{a}$ as the energy unit.

In the strongly-interacting limit, Hamiltonian $H$ can be mapped onto an anisotropic Heisenberg Hamiltonian thus realizing quantum magnetic phases 9, 10. In the following we focus on the Ising AF phase which, in bosonic language, is equivalent to a double checker-board (2CB) solid, characterized by broken $Z_{2}$ symmetry. To this end, we consider the hard-core boson regime set $t_{b}=0.15 t_{a}, U_{a b}=5.7 t_{a}$, so that the ground state of the homogenous system at half-integer filling corresponds to the $2 \mathrm{CB}$ phase [15]. We notice that the $2 \mathrm{CB}$ phase can be realized only for strong enough asymmetry between $t_{a}$ and $t_{b}$, i.e. if component $\mathrm{B}$ is, via e.g. optical depths tuning, effectively much heavier than A.

We have performed Quantum Monte Carlo simulations by means of the Worm Algorithm [19] of model $H$ for several curvatures of the harmonic potential $w / t_{a}=0.1$, $0.02,0.014,0.007,0.005,0.0025$, with maximum number of particles (corresponding to the most shallow trap) $N=N_{a}+N_{b} \sim 7200$. For each $w$ and $N$ the system size was chosen large enough so that no finite-size effects were present (ranging from linear size $L=60$ to $L=135$, depending on the specific case), and the temperature was fixed to $T=0.05 t_{a}$, well below the $2 \mathrm{CB}$ phase-transition temperature for the chosen Hamiltonian parameters [16. Our goal is to establish for which total particle number $N$ and population imbalance $\left(N_{a}-N_{b}\right)$ the $2 \mathrm{CB}$ phase is stabilized in the trap. To this extent we systematically analyze and classify different typologies of phase coexistence.

We have observed five distinct types of phase coexistence, as illustrated in Fig. 1. where the top (middle) row refers to the in trap density maps of the light (heavy) component, and the bottom row shows corresponding density profiles along a line going through the center of the trap. The color scheme in the top and middle rows is such that white (black) corresponds to density 1 (0). In the bottom row, dashed red, dotted blue and dot-dashed green refer to light component, heavy component and total density profiles, respectively. Each 
column is representative of an extended region in the plot of Fig. 2 summarizing the different types of phase coexistence found, as we shall discuss below. We classify the different typologies as follows (for each typology phases are listed as they appear going from the center to the edges of the trap):

2CB core: 2CB, light component superfluid (Fig. 1. panel a1-a2).

2CB ring: heavy component $\mathrm{MI}+$ light component superfluid, 2CB, light component superfluid (Fig. 1 . panel b1-b2).

global Mott core: global MI, i.e. a MI in the total density (see profile of total density, green line in Fig. 1 , panel c3 ), light component superfluid (see Fig. 1, panel c1-c2).

global Mott ring: heavy component MI + light component superfluid, global MI (see profile of total density, green line in Fig. 1, panel d3), light component superfluid (see Fig. 11, panel d1-d2).

phase separation: heavy component $\mathrm{MI}+$ light component superfluid, heavy component MI, light component SF (see Fig. 1, panel e1-e2).

For each of such sequences, subsequent phases are spatially separated by a thin shell of double superfluid. On the bottom row, density profiles make clear the choice of classification of typologies. In particular, in correspondence of the global MI phase (columns c) and d) ), we have observed corrugations in the density profiles of the single components, which have not completely disappeared within simulation time. We have observed that the super-counter-fluid phase [10] exhibits a similar behavior, and suspect that the global MI phase could display an analogous off-diagonal-long-range-order.

In all our simulations we have observed that the heavy component tends to locate itself at the center of the trap. This behavior can be understood as follows. In the strong-coupling regime considered here, delocalization of the heavy component (at the expense of the energy required to climb the walls of the trap) is suppressed compared with the delocalization of the light component, due to larger hopping parameter of the latter.

Our results for the three shallower trapping potentials simulated $\left(w=0.007,0.005,0.0025 t_{a}\right)$ are summarized in Fig. 2 where each symbol corresponds to one of the typologies described above (diamond for $2 \mathrm{CB}$ core, square for 2CB ring, downward triangle for global Mott core, upward triangle for global Mott ring, circle for phase separation). We can identify $\left(N_{a}, N_{b}\right)$ values (we scale the number of particles by the curvature of the trapping potential, see below) for which the $2 \mathrm{CB}$ phase is realized in certain regions of the trap: squares and diamonds, corresponding to $2 \mathrm{CB}$ ring and $2 \mathrm{CB}$ core, respectively. We note that, in general, the $2 \mathrm{CB}$ phase can be observed only if the light component is the majority one, and provided $w<0.01 t_{a}$. We have studied larger values of $w\left(=0.014,0.02,0.1 t_{a}\right)$, i.e. steeper traps, and have not observed $\mathrm{CB}$ for any $\left(N_{a}, N_{b}\right)$. In other words, the trapping potential should

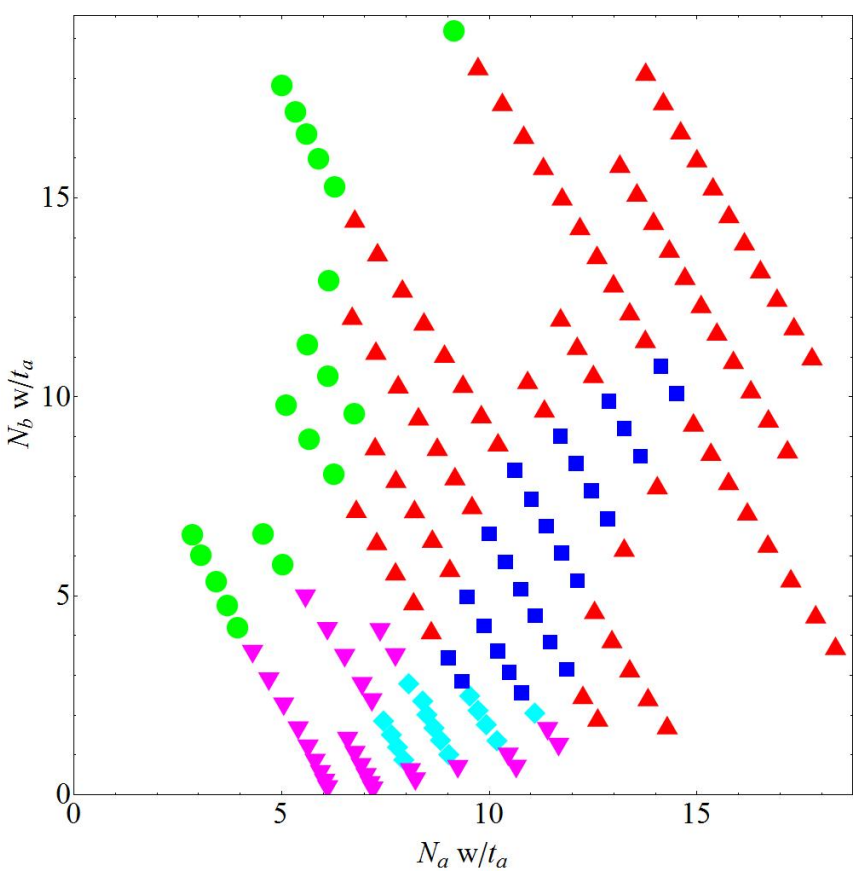

FIG. 2: (Color online) Typologies of phase coexistence (see text) as a function of number of particles $N_{a}, N_{b}$ scaled by the trap curvature for $w=0.007,0.005,0.0025 t_{a}$. Diamonds: CB core; squares: $\mathrm{CB}$ ring; downward triangles : global Mott core; upward triangles: global Mott ring; circles: phase separation. Error bars are much smaller than symbol size (maximum percent error is $0.5 \%$ ).

be smooth enough to allow stabilization of $2 \mathrm{CB}$ ring or core regions. For $w=0.007,0.005,0.0025 t_{a}$, instead, we can summarize our results on the same plot by using $w N_{a} / t_{a}$ and $w N_{b} / t_{a}$ as axis (the smoother the trap, the wider $2 \mathrm{CB}$ core and ring regions are). Upon further decreasing the trap curvature $w$ we have observed that $2 \mathrm{CB}$ ring region in the $w N_{a} / t_{a}$ vs $w N_{b} / t_{a}$ plane tends to grow, although extremely slowly (e.g. lowering $w$ by a factor of 2, would only convert a single upward triangle into a square, i. e. a global MI ring would become a CB ring, in the plot of Fig. 2). In other words, for much shallower trap curvatures, the $\mathrm{CB}$ ring region would be only slightly larger than the one in the plot of Fig. 2 (i. e. the system exhibits a scalable behavior for a wide range of $w$ 's). Hence, Fig. 2 can be used as guidance for experiments since it provides $N_{a}$ and $N_{b}$ required for the observation of the Ising AF for experimentally realizable trap curvatures.

Although the hard-core limit corresponds to most stable quantum magnetic phases against hopping [9], we will now relax this constraint. We have performed simulations at finite intraspecies interaction $U_{a}=U_{b}=U$ both in uniform and trapped system. Our goal is to probe how far one can soften $U$ for given values of hopping parameters $t_{a}, t_{b}$, and interspecies interaction $U_{a b}$. We have found that for $t_{b}=0.15 t_{a}$ and $U_{a b}=5.7 t_{a}$ 
the $2 \mathrm{CB}$ phase in a uniform system survives down to $U \sim 70 t_{a}$, i.e. $U / U_{a b} \simeq 12$. If we further increase the hopping asymmetry, e.g. $t_{b}=0.075 t_{a}$, a $2 \mathrm{CB}$ phase still exists at $U \sim 40 t_{a}$, i.e. $U / U_{a b} \simeq 7$. We expect that the extension and position of $2 \mathrm{CB}$ regions in Fig 2 will slightly change upon relaxing the hard-core constraint. In particular, we expect the $2 \mathrm{CB}$ regions to shrink (due to a more fragile CB phase for soft-core atoms) as phase separation becomes more favorable. Preliminary numerical results agree with such an expectation but also suggest that the general qualitative results (i.e. typologies observed) valid for hard-core bosons should still be valid. More specifically, we have performed simulations in a trap with parameters $w / t_{a}=0.005$, $t_{b}=0.075 t_{a}, U_{a b}=5.7 t_{a}, U=40 t_{a}, N_{a}=1240$ and $N_{b}=170$ and found that the $2 \mathrm{CB}$ core still exist.

A previous mean-field study carried on for soft-core bosonic atoms claimed realization of $2 \mathrm{CB}$ ring and core regions in a trap 20]. The parameters used in 20] are the following: $w / t_{a}=0.064, t_{b}=0.16 t_{a}, U_{a b}=5.7 t_{a}$, $U_{a}=1.9 t_{a}, U_{b}=37.5 t_{b}$. We note that component A is rather soft. Preliminary numerical simulations with the same parameters indicate that phase separation will occur both, with and without the harmonic trapping potential (in the presence of the latter complete phase separation, i.e. no overlap between the two components anywhere in the trap, seems to occur). Moreover, our numerical results for soft-core atoms in uniform systems, indicate that the $2 \mathrm{CB}$ phase will be destroyed upon softening $U<70 t_{a}$ with $t_{b}=0.15 t_{a}$. Hence, the results of 20] seem to be rather controversial.

Finally, we would like to provide estimates for experimental setups required for the observation of the Ising AF (we use a harmonic approximation around the minima of the optical lattice potential [21]). The hard-core limit can be achieved by realizing $\mathrm{a}_{\alpha} \gg \mathrm{a}_{a b}$ $(\alpha=a, b)$, with $\mathrm{a}_{\alpha}$ and $\mathrm{a}_{a b}$ intra- and interspecies scattering lengths, respectively. The latter inequalities can be satisfied by e.g. making use of a combination of $\mathrm{dc}$ and rf magnetic fields [22], or dc electric fields [23] in order to independently tune two scattering lengths via Feshbach resonances. Alternatively, for what concerns $U_{a b}$ tuning, Wannier-function overlap in the presence of state-dependent lattices can be manipulated. Harmonic frequencies for the trapping potential are given by $\omega_{\alpha}^{2}=2 w /\left(m_{\alpha} a^{2}\right)$. Let us consider laser beams with $\lambda=1064 \mathrm{~nm}$ and a mixture of ${ }^{87} \mathrm{Rb}$ atoms in hyperfine states $|1,-1\rangle$ (A component) and $|2,-2\rangle$ (B component), for which $\mathrm{a}_{a b}=98.09 a_{0}$ ( $a_{0}$ is the Bohr radius), $\mathrm{a}_{a}=100.4 a_{0}$ and $\mathrm{a}_{b}=98.98 a_{0}$. According to the choice in 24, with a harmonic confinement of $\omega_{a}=\omega_{b} \sim 2 \pi 370 \mathrm{~Hz}$ (corresponding to $w=0.005 t_{a}$ ) one can observe the $2 \mathrm{CB}$ core (ring) by loading $N_{a} \sim 1700$ (2200), $N_{b} \sim 300$ (800). With $\omega_{a}=\omega_{b} \sim 2 \pi 250 \mathrm{~Hz}$ (corresponding to $w=0.0025 t_{a}$ ), instead, one can observe the $2 \mathrm{CB}$ core (ring) by loading $N_{a} \sim 3400$ (4400), $N_{b} \sim 600$ (1600). For shallower traps, e.g. $\omega \sim 2 \pi 80 \mathrm{~Hz}$, one should load $N_{a} \sim 34000$ (44000) and $N_{b} \sim 6000$ (16000).

In the case of ${ }^{87} \mathrm{Rb}-{ }^{41} \mathrm{~K}$ mixtures (we choose ${ }^{87} \mathrm{Rb}$ as the effectively-light component i. e. component A) one has $\mathrm{a}_{\mathrm{RbK}}=163 a_{0}, \mathrm{a}_{\mathrm{Rb}}=99 a_{0}$ and $\mathrm{a}_{\mathrm{K}}=65 a_{0}$. According to the choice in [25], with a harmonic confinement of $\omega_{\mathrm{Rb}} \sim 2 \pi 366 \mathrm{~Hz}$ and $\omega_{\mathrm{K}} \sim 2 \pi 525 \mathrm{~Hz}$ (corresponding to $w=0.005 t_{a}$ ), the $2 \mathrm{CB}$ core (ring) is realized by loading $N_{\mathrm{Rb}} \sim 1700(2200), N_{\mathrm{K}} \sim 300$ (800). With a choice of $\omega_{\mathrm{Rb}} \sim 2 \pi 254 \mathrm{~Hz}$ and $\omega_{\mathrm{K}} \sim 2 \pi 381 \mathrm{~Hz}$ (corresponding to $w=0.0025 t_{a}$ ), the $2 \mathrm{CB}$ core (ring) is realized by loading $N_{\mathrm{Rb}} \sim 3400$ (4400), $N_{\mathrm{K}} \sim 600$ (1600).

In conclusion, we have presented accurate results based on Quantum Monte Carlo simulations of twocomponent bosonic systems on a square lattice and in the presence of an external harmonic confinement. Starting from hopping parameters and interaction strengths which stabilize the Ising antiferromagnetic in the homogeneous case and at half integer filling factor, we have studied how the presence of the harmonic confinement challenges the realization of such phase. We have considered realistic trapping frequencies and number of particles, and established under which conditions, i.e. total number of particles and population imbalance, the antiferromagnetic phase can be observed in the trap. With recently developed single atom imaging techniques, direct observation of the Ising ferromagnetic phase is possible [17, 18. Alternatively one can use Bragg spectroscopy in order to probe density-density correlations [27. Given the challengingly low-temperature scale associated to quantum magnetic phases, it would be certainly interesting to study signatures of the onset quantum magnetic phases on the approach of the critical temperature from above.

We would like to thank F. Minardi, S. Söyler and S. Kuhr for fruitful discussions. This work was supported by the Institute for Atomic, Molecular and Optical Physics (ITAMP). MG wants to thank ITAMP for warm hospitality and financial support.
[1] S. Ospelkaus et al., Phys. Rev. Lett. 96, 180403 (2006).

[2] K. Günter et al., Phys. Rev. Lett. 96, 180402 (2006).

[3] I. Bloch, J. Dalibard, W. Zwerger, Rev. Mod. Phys. 80, 885, (2008).

[4] Th. Best et al., Phys. Rev. Lett. 102, 030408 (2009).

[5] J. Catani et al., Phys. Rev. A 77, 011603(R) (2008).
[6] B. Gadway et al., Phys.Rev.Lett. 105, 045303 (2010).

[7] D. M. Weld et al., Phys. Rev. Lett. 103, 245301 (2009).

[8] D. M. Weld et al., Phys. Rev. A 82, 051603(R) (2010).

[9] E. Altman et al., New J. Phys. 5, 113 (2003).

[10] A.B. Kuklov and B.V. Svistunov, Phys. Rev. Lett. 90, 100401 (2003). 
[11] K. Miyake, S. Schmitt-Rink, and C. M. Varma, Phys. Rev. B 34, 6554 (1986); D. J. Scalapino, E. Loh, and J. E. Hirsch, Phys. Rev. B 34, 8190 (1986); P. Monthoux, D. Pines, and G. G. Lonzarich, Nature 450, 1177 (2007).

[12] O. Stockert1 et al., Nat. Phys. 7, 119 (2011).

[13] J. Simon et al. Nature 472, 307 (2011)

[14] S. Pielawa et al., arXiv:1101.2897.

[15] G. Söyler et al., New J. Phys. 11, 073036 (2009).

[16] B. Capogrosso-Sansone et al., Phys. Rev. A 81, 053622 (2010).

[17] W. S. Bakr et al., Nature, 462, 74 (2009); W. S. Bakr et al., Science 329, 547 (2010).

[18] J. F. Sherson et al., Nature 467, 68 (2010); C. Weitenberg et al., Nature 471, 319 (2011).

[19] N. V. Prokof'ev, B. V. Svistunov, and I. S. Tupiysyn, Phys. Lett. A 238, 253 (1998); Sov. Phys. JETP 87, 310 (1998).

[20] U. Shrestha, Phys. Rev. A 82, 041603(R) (2010).
[21] F. Gerbier et al., Phis. Rev. A 72, 053606 (2005).

[22] T. V. Tscherbul et al., Phys. Rev. A 81, 050701(R) (2010).

[23] R. V. Krems, Phys. Rev. Lett. 96, 123202 (2006).

[24] If lattice depths (in units of recoil energy) are $s_{a} \sim 16$, $s_{b} \sim 27$, one obtains $t_{b} \simeq 0.15 t_{a}, U_{a a} \simeq 70 t_{a}, U_{b b} \simeq 100 t_{a}$ $\left(U_{b b} \simeq 667 t_{b}\right), t_{a} \simeq 11.6 \mathrm{~Hz}$. With a shift between state dependent lattices of $\delta x / d=0.345$ one has $U_{a b} \simeq 5.7 t_{a}$.

[25] If $s_{\mathrm{Rb}} \sim 16, s_{\mathrm{K}} \sim 31$ (in units of the respective recoil energies) and scattering length $a_{s}^{(\mathrm{Rb} \mathrm{K})}$ is tuned via Feshbach resonance [26] to $4.1 a_{0}$ one obtains $t_{b} \simeq 0.15 t_{a}$, $U_{a b} \simeq 5.7 t_{a}, U_{a a} \simeq 70 t_{a}, U_{b b} \simeq 1064 t_{b}\left(U_{b b} \simeq 160 t_{a}\right)$, $t_{a} \simeq 11.5 \mathrm{~Hz}$. Alternatively, instead of tuning $a_{s}^{(\mathrm{Rb} \mathrm{K})}$ one could shift the two lattices by $\delta x / a=0.40$.

[26] G. Thalhammer et al., Phys. Rev. Lett. 100, 210402 (2008).

[27] D. Clément et al., J. Low Temp.Phys 158, 5 (2010). 